\title{
Structural vacancies in (Ti,Al)N: An ab initio study
}

\author{
Axel Forslund $\oplus^{1}$ and Andrei V. Ruban ${ }^{1,2}$ \\ ${ }^{1}$ Department of Materials Science and Engineering, KTH Royal Institute of Technology, SE-100 44 Stockholm, Sweden \\ ${ }^{2}$ Materials Center Leoben Forschung GmbH, A-8700 Leoben, Austria
}

(Received 14 September 2021; revised 27 October 2021; accepted 11 January 2022; published 21 January 2022)

\begin{abstract}
The zero K formation energies of metal and nitrogen vacancies in several (Ti,Al)N alloys and at the (001) $(\mathrm{Ti}, \mathrm{Al}) \mathrm{N} / \mathrm{AlN}$ interface are obtained in ab initio supercell calculations. The dependence of the formation energies of metal vacancies on their local environment and type are analyzed and explained in terms of effective cluster interactions for unrelaxed calculations. The common trend for all investigated types of vacancies is that their formation energy increases with the number of $\mathrm{Al}$ nearest neighbors if local lattice relaxations are not allowed. However, local lattice relaxations produce a dramatic effect especially in the case of metal vacancies leading to a complicated nonlinear dependence on the local environment indicating the existence of strong multisite strain-induced interactions.
\end{abstract}

DOI: 10.1103/PhysRevMaterials.6.013803

\section{INTRODUCTION}

Titanium nitride and titanium-aluminum nitride films are used as hard coatings for cutting tools due to their exceptional mechanical and tribological properties [1-3]. They are produced using either physical or chemical vapor deposition (PVD or CVD) and the growth process strongly affects their composition and structure at the atomic level. In particular, the usually formed B1 structure with fcc-like Ti (Al) and N sublattices often contains nonequilibrium structural defects. The most easily formed are vacancies on both sublattices, although the formation of interstitials and complexes is possible. As has recently been demonstrated [4], the amount of vacancies can be controlled in reactive PVD by varying $\mathrm{N}_{2}$ pressure (or chemical potential) during the film growth and that allows one to significantly reduce their amount and thereby increase the thermal stability of metastable (Ti,Al)N alloys.

In this paper, we investigate the energetics of structural $\mathrm{Ti}(\mathrm{Al})$ and $\mathrm{N}$ vacancies in (Ti, $\mathrm{Al}) \mathrm{N}$ random alloys for the following compositions: $\mathrm{TiN},\left(\mathrm{Ti}_{0.5} \mathrm{Al}_{0.5}\right) \mathrm{N},\left(\mathrm{Ti}_{0.125} \mathrm{Al}_{0.875}\right) \mathrm{N}$, $\left(\mathrm{Ti}_{0.083 .} . \mathrm{Al}_{0.916 .}\right) \mathrm{N}$, and $\mathrm{B} 1-\mathrm{AlN}$. These compositions are in the range of those which have been observed in $(\mathrm{Ti}, \mathrm{Al}) \mathrm{N}$ lamella structures produced by CVD [5]. In fact, we closely follow the earlier $a b$ initio investigation of the metal and nitrogen vacancy formation energetics by Euchner and Mayrhofer [6] but using a larger setup for supercell models of alloys and investigating the details of an element and local environment specific of the vacancy formation energies similar to the investigation by Tasnadi et al. [7].

Published by the American Physical Society under the terms of the Creative Commons Attribution 4.0 International license. Further distribution of this work must maintain attribution to the author(s) and the published article's title, journal citation, and DOI. Funded by Bibsam.
Structural vacancies in B1 IV-group carbides and nitrides have been in the focus of theoretical and experimental studies for decades. Usually, structural vacancies are present on the nitrogen or carbide sublattice, where at low temperatures they form ordered structures at different temperatures and compositions [8]. This is also the case of $\mathrm{B}^{-}-\mathrm{TiN}_{x}$ with nitrogen deficient compositions where several B1-TiN based ordered alloys $\left(\mathrm{Ti}_{2} \mathrm{~N}_{3}, \mathrm{Ti}_{4} \mathrm{~N}_{3}\right.$, and $\left.\mathrm{Ti}_{6} \mathrm{~N}_{5}\right)$ are stable according to an $a b$ initio investigation by $\mathrm{Yu}$ et al. [9].

In this respect, B1-AIN exhibits a completely different behavior due to the fact that it is an insulator, and thus formation of any point defect is highly energetically costly, if it changes the ionic balance. An addition of $\mathrm{Al}$ to TiN also leads to an increase of the formation energy of the structural nitrogen vacancies as has been demonstrated in ab initio calculations $[6,10]$. At the same time, the energetics of metal vacancies in random $(\mathrm{Ti}, \mathrm{Al}) \mathrm{N}$ alloys seems to be more complicated due to strong local environment effects [7]. This is the main subject of the present theoretical investigation together with the effect of the alloy composition.

We determine the formation energies of structural vacancies, i.e., the energy of removing a particular element from the system. The calculations are done using idealized model systems represented by finite supercells. Therefore, the connection between the present results to the PVD- or CVDgrown real systems is indirect and, especially considering the structural and spatial complexity of the real systems, a clear interpretation of the results is hard for any finite supercell model. Nevertheless, the detailed information on local environment obtained here can be useful for future atomistic modeling of these processes.

\section{DETAILS OF AB INITIO CALCULATIONS}

\section{A. Supercell projector augmented wave (PAW) calculations}

The (Ti-Al)N alloys were modeled by supercells of different sizes, whose electronic structure and total 
energy were calculated assuming translational symmetry. The supercell sizes were 216 sites $[3 \times 3 \times 3(\times 8)]$ for TiN, AlN, and $\left(\mathrm{Ti}_{0.5} \mathrm{Al}_{0.5}\right) \mathrm{N}$ and 384 sites $[4 \times 4 \times 3(\times 8)]$ for $\left(\mathrm{Ti}_{0.125} \mathrm{Al}_{0.875}\right) \mathrm{N}$ and $\left(\mathrm{Ti}_{0.083} \ldots \mathrm{Al}_{0.916 \ldots}\right) \mathrm{N}$. Some calculations for $\left(\mathrm{Ti}_{0.5} \mathrm{Al}_{0.5}\right) \mathrm{N}$ were also done using a 512-atom $4 \times 4 \times 4(\times 8)$ supercell. These supercells were built upon the initial cubic B1 unit cell comprising four metal and four nitrogen atoms. The positions of the $\mathrm{Ti}$ and $\mathrm{Al}$ atoms in the case of Ti-Al alloys on the metal sublattice were chosen by minimizing the Warren-Cowley short-range order parameters at the first eight coordination shells of the underlying fcc lattice. In the case of $\left(\mathrm{Ti}_{0.5} \mathrm{Al}_{0.5}\right) \mathrm{N}$ random alloy, several three- and four-site atomic distribution correlation functions were also optimized to be close to those in the corresponding completely random alloy.

The electronic structure and total energy calculations were done by the projector augmented wave (PAW) method [11] as implemented in the Vienna ab initio simulation package (VASP) [12-14]. All the PAW calculations were done using the PBE exchange correlation potential and energy [15]. The so-called "Ti-sv," "Al," and "N-s" PAW potentials were used in all the calculations. The kinetic energy cutoff was $400 \mathrm{eV}$. The integration over the Brillouin zone was performed using $4 \times 4 \times 4$ and $4 \times 4 \times 6$ grids of $k$ points for the 512and 384-atom supercells, respectively, determined according to the Monkhorst-Pack scheme [16]. The $k$-point grid was appropriately rescaled if the supercell size was different (in the case of TiN and AlN). In all the PAW calculations, the shape of the supercell was fixed in order to keep the underlying B1 structure cubic on average. The lattice constants were 4.25, 4.11, 4.10, 4.09, and $4.07 \AA$ for $\mathrm{TiN},\left(\mathrm{Ti}_{0.5} \mathrm{Al}_{0.5}\right) \mathrm{N}$, $\left(\mathrm{Ti}_{0.125} \mathrm{Al}_{0.875}\right) \mathrm{N},\left(\mathrm{Ti}_{0.083} . . \mathrm{Al}_{0.916 \ldots}\right) \mathrm{N}$, and $\mathrm{AlN}$, respectively. Only local atomic positions were relaxed in the PAW-VASP calculations.

\section{B. Effective interactions calculations}

In order to describe local environment effects in (Ti,Al)N alloys upon the formation energy of structural vacancies, we also calculated the effective pair interactions in the $\left(\mathrm{Ti}_{0.49} \mathrm{Al}_{0.49} \mathrm{Va}_{0.02}\right) \mathrm{N}$ alloy using the screened generalized perturbation method (SGPM) [17-21], as was implemented in the Lyngby version [22] of the exact muffin-tin orbital (EMTO) method [23,24]. In these calculations, the electronic structure of random Ti-Al alloys on the metal sublattice was obtained using the coherent potential approximation (CPA) [25-27].

The intrasite Coulomb interactions and intersite screening constants $[19,20]$ were obtained in the supercell calculations by the locally self-consistent Green's function (LSGF) $[28,29]$ method implemented within the EMTO technique (ELSGF) [30], which accurately accounts for local environment effects in the electronic structure of alloys. The screening parameters for the $\left(\mathrm{Ti}_{0.49} \mathrm{Al}_{0.49} \mathrm{Va}_{0.02}\right) \mathrm{N}$ alloy were determined in 432atom $[6 \times 6 \times 6(\times 2)]$ calculations with an equal number of empty spheres to provide a better packing condition for the atomic spheres in the EMTO method. All the self-consistent EMTO-CPA and ELSGF calculations were performed with the orbital momentum cutoff of $l_{\max }=3$ for partial waves. The integration over the Brillouin zone was performed using a $21 \times 21 \times 21$ grid of $k$ points for the fcc-based B1 unit

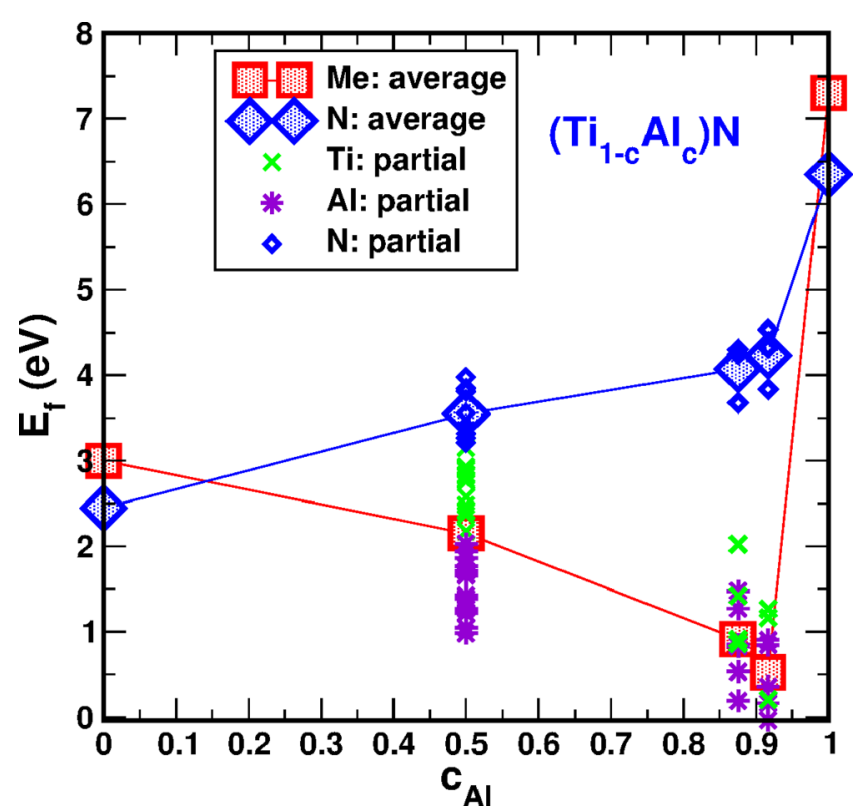

FIG. 1. Vacancy formation energies in $\mathrm{B} 1 \mathrm{TiN}$, $\mathrm{AlN}$, $\left(\mathrm{Ti}_{0.5} \mathrm{Al}_{0.5}\right) \mathrm{N}, \quad\left(\mathrm{Ti}_{0.125} \mathrm{Al}_{0.875}\right) \mathrm{N}, \quad$ and $\quad\left(\mathrm{Ti}_{0.083 \ldots} \mathrm{Al}_{0.916 \ldots}\right) \mathrm{N}, \quad$ from relaxed calculations.

cell. Self-consistent electron densities were obtained within the local density approximation (LDA) [31]. The calculated on-site screening parameters are $0.73,0.74$, and 0.64 for $\mathrm{Ti}$, $\mathrm{Al}$, and $\mathrm{Va}$, correspondingly.

\section{RESULTS}

\section{A. Structural vacancy formation energies: PAW supercell results}

The vacancy formation energy has been calculated as the energy difference between the supercell with and without vacancy plus the energy of the vacant element in its ground state structure:

$$
E_{\mathrm{vac}}^{i}=E_{\mathrm{tot}}^{i}-E_{\mathrm{tot}}^{0}+E_{i} .
$$

Here, $E_{\mathrm{vac}}^{i}$ is the structural vacancy formation energy of element $i$ and $E_{\mathrm{tot}}^{i}, E_{\mathrm{tot}}^{0}$, and $E_{i}$ the total energies of the supercell with vacancy, without vacancy, and the ground state energy of element $i$ (per atom). In general, to relate the vacancy formation energies to experiment, one should, of course, use reference energies corresponding to the experimental system in such a definition. Here, it is for convenience defined in the usually accepted way, where the ground state energies are calculated for fcc $\mathrm{Al}$, hcp Ti, and the $\mathrm{N}_{2}$ molecule.

In the case of random alloys, the vacancy formation energy becomes dependent on the particular alloy configuration as well as on the local configuration of alloy components next to the vacancy. The strongest one is usually associated with the number of $\mathrm{Ti}$ or $\mathrm{Al}$ atoms at the first couple of coordination shells around a vacancy.

In Fig. 1, we show our results for the structural Ti, Al, and $\mathrm{N}$ vacancies in the considered alloys from relaxed calculations. In the case of alloys, the average vacancy formation energies are shown by large square and diamond symbols for the metal and nitrogen vacancies, respectively. They are 


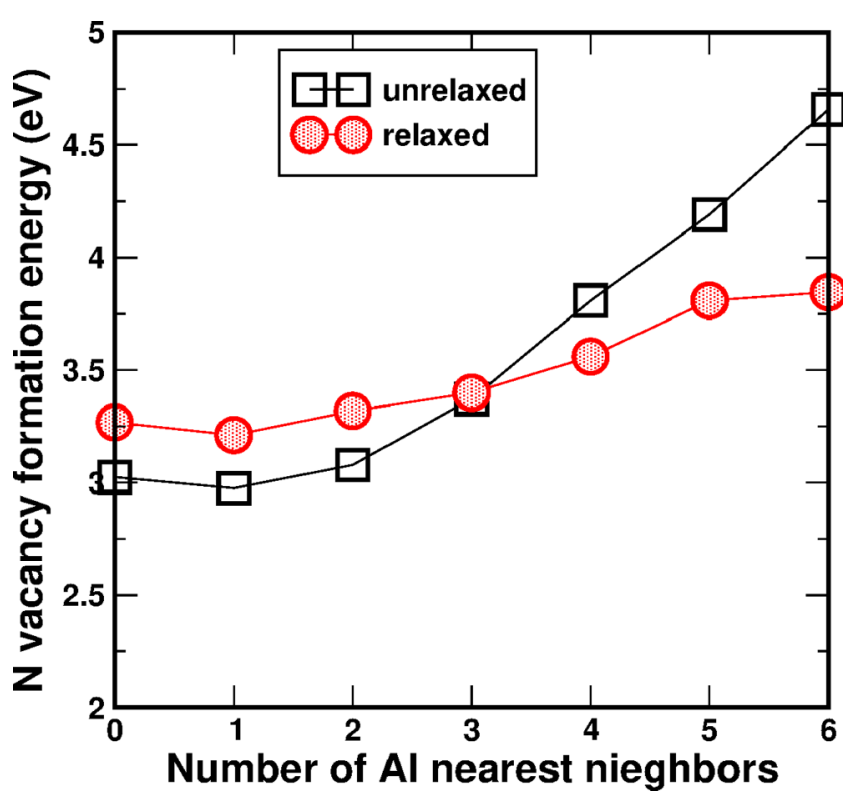

FIG. 2. Nitrogen vacancy formation energy in $\left(\mathrm{Ti}_{0.5} \mathrm{Al}_{0.5}\right) \mathrm{N}$ as a function of the number of $\mathrm{Al}$ atoms next to a vacancy. [The number of $\mathrm{Al}$ atoms at the second coordination shell of the metal lattice is four (and four Ti atoms) in all the cases, while the number of $\mathrm{Al}$ atoms at the third coordination shell varies between 10 and 14 (there are 24 sites at the third metal coordination shell).]

obtained after simple averaging of the local contributions shown by crosses, stars, and small diamond symbols, i.e., without taking into consideration the probability to have a particular local environment in a random alloy, and therefore these energies are mostly for demonstrating trends.

The choice of the local vacant sites was dictated by maximizing the variation of the number of the nearest neighbors of $\mathrm{Al}$ (or $\mathrm{Ti}$ ) atoms including possible variations of the number of $\mathrm{Al}(\mathrm{Ti})$ atoms at the second coordination shell of the metal sublattice. Obviously, the case of the $\left(\mathrm{Ti}_{0.5} \mathrm{Al}_{0.5}\right) \mathrm{N}$ supercell provides the largest number of sites with such variations, which we use here to demonstrate the effect of local environment on the vacancy formation energies.

In Fig. 2, we show the dependence of the nitrogen vacancy formation energy on the number of $\mathrm{Al}$ atoms next to the vacant site for the case of relaxed and unrelaxed calculations (with a fixed 50:50 composition at the second coordination shell). In the former case only local lattice relaxations were taken into consideration, while in the case of unrelaxed calculations, both supercells, with and without vacancies, were calculated assuming ideal lattice positions of the B1 underlying structure. Thus unrelaxed vacancy formation energies present just a "chemical" contribution to the total vacancy formation energy.

Clearly, the chemical part of the vacancy formation energy increases with the number of $\mathrm{Al}$ atoms next to the vacancy, which is an expected trend due to the fact there is usually an abundance of the structural nitrogen vacancies in TiN. At the same time, the local lattice relaxations significantly flatten such a dependence, and their contribution is especially strong when the nitrogen vacancy is surrounded only by $\mathrm{Al}$ atoms. The formation energies of the nitrogen vacancy increase with

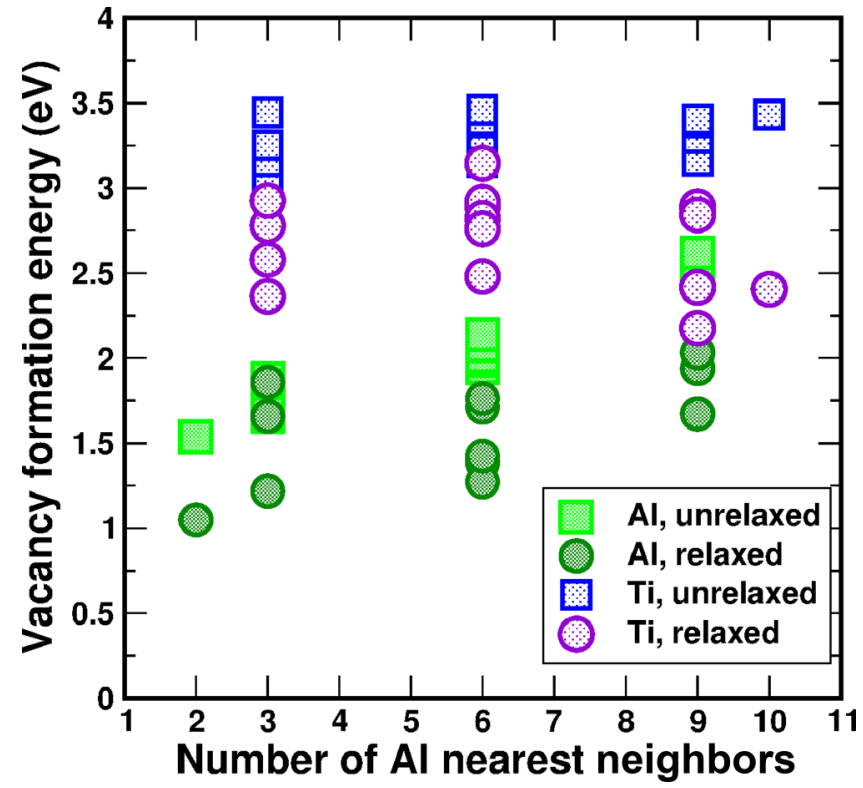

FIG. 3. $\mathrm{Al}$ and $\mathrm{Ti}$ vacancy formation energies in $\left(\mathrm{Ti}_{0.5} \mathrm{Al}_{0.5}\right) \mathrm{N}$ as a function of the number of $\mathrm{Al}$ atoms in the first coordination shell, $n_{1}^{\mathrm{Al}}$.

Al concentration in $\left(\mathrm{Ti}_{1-c} \mathrm{Al}_{c}\right) \mathrm{N}$ alloys and our results are in good agreement with the results of Euchner et al. [6].

Although we have not thoroughly investigated the effect of the local compositions of the other coordination shells on the nitrogen vacancy formation energy, several estimates show that it is much less significant than that of the first coordination shell. At the same time, the situation becomes much more complicated in the case of metal vacancies as has been discovered and discussed already by Tasnadi et al. [7].

In Fig. 3, we show the $\mathrm{Al}$ and $\mathrm{Ti}$ vacancy formation energies in $\left(\mathrm{Ti}_{0.5} \mathrm{Al}_{0.5}\right) \mathrm{N}$ as a function of the number of $\mathrm{Al}$ atoms at the first coordination shell of the metal sublattice, $n_{1}^{\mathrm{Al}}$. Also here we show the results of the unrelaxed and relaxed calculations to separate out the "chemical" contribution. The latter shows a very clear difference of the dependence of $\mathrm{Al}$ and Ti vacancy formation energies on the local environment. While the formation energies of Ti vacancies do not show a clear dependence on the number of $\mathrm{Al}$ nearest neighbors, the $\mathrm{Al}$ vacancy formation energies grow substantially with $n_{1}^{\mathrm{Al}}$.

This to some extent counterintuitive result will be considered in the next section, but here we would like to point out that, obviously, local environment effects beyond the first coordination shell are important and they can be quite complicated and strong, especially due to local lattice relaxations which can change the vacancy formation energy by more than $1 \mathrm{eV}$.

Such strong local environment effects are clearly seen in Fig. 1 as widely dispersed results. In the case of $\left(\mathrm{Ti}_{0.125} \mathrm{Al}_{0.875}\right) \mathrm{N}$ and $\left(\mathrm{Ti}_{0.083 . .} \mathrm{Al}_{0.916 . .}\right) \mathrm{N}$ alloys, the spread of the formation energies would most probably be the same, but it is difficult to find sites with different configurations in the relatively small supercell when the alloy composition is far from equiatomic.

Let us note that our results for these Al-rich alloys are at variance with the previous calculations by Euchner et al. 
[6]: while in their calculations the vacancy formation energy of metal atoms increases quite sharply with $\mathrm{Al}$ concentration when $c_{\mathrm{Al}}>0.8$, it continues to decrease in our calculations and then it becomes very large in the B1 ordered AlN, which is again in agreement with Ref. [6].

We do not know the origin of this dramatic difference in the results. The reason why the vacancy formation energies are so high in the B1-AlN is clear: as has been already mentioned, it is almost an ionic compound. At the same time, both $\left(\mathrm{Ti}_{0.125} \mathrm{Al}_{0.875}\right) \mathrm{N}$ and $\left(\mathrm{Ti}_{0.083} \ldots \mathrm{Al}_{0.916 \ldots} . . \mathrm{N}\right.$ come out as metal systems in our calculations. Thus such a dramatic change of the vacancy formation energies is most probably associated with the change of the type of the bonding.

\section{B. Configurational dependence of the formation energies of metal vacancies}

In this section, we would like to make a connection between the configurational dependencies of the $\mathrm{Al}$ and $\mathrm{Ti}$ vacancy formation energies (in particular, their dependence on the number of the $\mathrm{Al}$ atoms at the first coordination shell of the vacant site) and effective interactions. For that purpose we use the following configurational Hamiltonian which is consistent with SGPM effective interactions, which contain only the "chemical" contribution and can easily by determined for unrelaxed lattices:

$$
\begin{aligned}
H= & \frac{1}{2} \sum_{p} \sum_{\alpha, \beta \neq \delta} V_{p}^{(2) ; \alpha \beta[\delta]} \sum_{i j \in p} \delta c_{i}^{\alpha} \delta c_{j}^{\beta} \\
& +\frac{1}{3} \sum_{t} \sum_{\alpha, \beta, \gamma \neq \delta} V_{t}^{(3) ; \alpha \beta \gamma[\delta]} \sum_{i, j, k} \delta c_{i}^{\alpha} \delta c_{j}^{\beta} \delta c_{k}^{\gamma}+\text { h.o.t. }
\end{aligned}
$$

The summation is performed over different types of clusters ( $p$ and $t$ stand for indexes of pairs and triangles), alloy components (designated by Greek letters, where $\delta$ is for the "dependent component"), and lattice sites $(i, j$, and $k)$; $V_{p}^{(2) ; \alpha \beta[\delta]}$ and $V_{t}^{(3) ; \alpha \beta \gamma[\delta]}$ are the pair- and three-site effective concentration and volume dependent interactions; $\delta c_{i}^{\alpha}=c_{i}^{\alpha}-$ $c^{\alpha}$ is the concentration fluctuation of the $\alpha$ component at site $i$ from its average concentration in the alloy, $c^{\alpha}$. In our case, we should deal with a three-component alloy, i.e., $\alpha, \beta, \gamma$, and $\delta$ can be $\mathrm{Ti}, \mathrm{Al}$, or $\mathrm{Va}$ (vacancy).

The contribution from pair interactions in (2) can be reduced to a convenient quasibinary form, which allows one to single out contributions from different alloy pairs and make a clear picture of configurational trends in multicomponent alloys [22]:

$$
H^{(2)}=-\frac{1}{2} \sum_{p} \sum_{\alpha \neq \beta} V_{p}^{(2) ; \alpha \beta} \sum_{i j \in p} \delta c_{i}^{\alpha} \delta c_{j}^{\beta} .
$$

Here, $V_{p}^{(2) ; \alpha \beta}$ are the usual binary effective interactions describing the mutual ordering of $\alpha$ and $\beta$ atoms and are related to the multicomponent effective pair interactions $V_{p}^{(2) ; \alpha \beta[\delta]}$ as [32]

$$
V_{p}^{(2) ; \alpha \beta[\delta]}=\frac{1}{2}\left[V_{p}^{(2) ; \alpha \delta}+V_{p}^{(2) ; \beta \delta}-V_{p}^{(2) ; \alpha \beta}\right] .
$$

In the following derivation, we will use the quasibinary form of the configurational Hamiltonian (3) in order to address the origin of different dependences of the $\mathrm{Ti}$ and $\mathrm{Al}$ vacancy

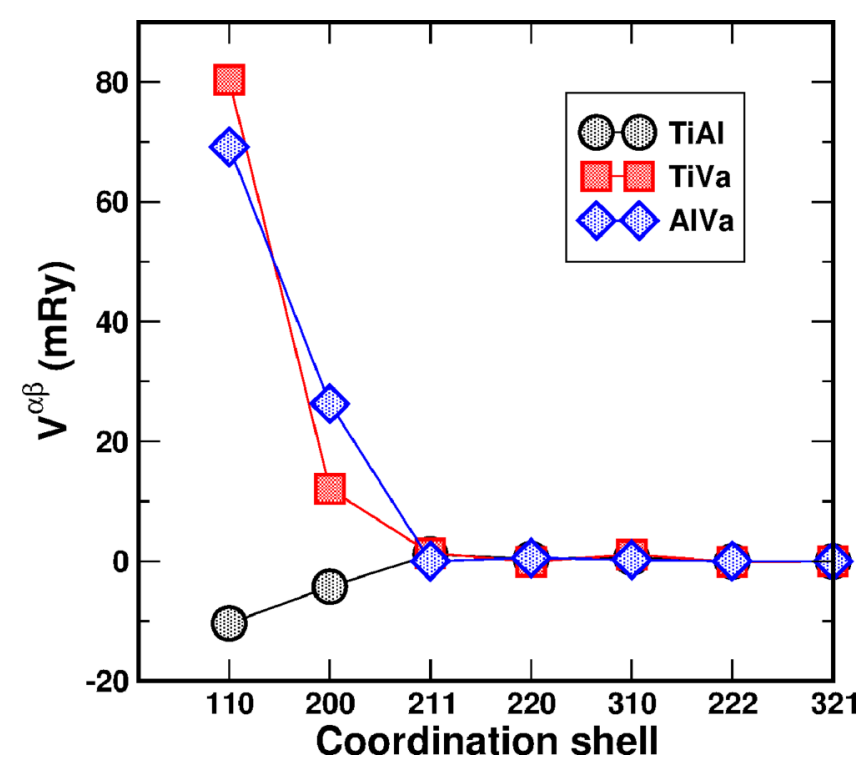

FIG. 4. Effective Ti-Al, Ti-Va, and Al-Va interactions obtained by the SGPM in the EMTO-CPA calculations of the $\left(\mathrm{Ti}_{0.49} \mathrm{Al}_{0.49} \mathrm{Va}_{0.02}\right) \mathrm{N}$ alloy for different neighbor coordinates.

formation energies on the number of nearest neighbor $\mathrm{Al}$ (or Ti) atoms as are clearly seen in Fig. 3. In this case, $\delta c_{i}^{\alpha}$ can equivalently be substituted by concentration variables $c_{i}^{\alpha}$, which allows one to simplify the derivation for the change of the energy of the system due to removing, for instance, an $\mathrm{Al}$ atom from a particular site (or creating an $\mathrm{Al}$ vacancy).

The contribution from the effective pair interactions at coordination $i, V_{i}^{\alpha \beta}$, to the vacancy formation energy of $\mathrm{Al}$ having $n_{i}^{\mathrm{Al}} \mathrm{Al}$ atoms at this coordination shell is

$$
\begin{aligned}
E^{\mathrm{Al}}\left(n_{i}^{\mathrm{Al}}\right)= & -\frac{1}{2}\left[V_{i}^{\mathrm{TiVa}}\left(z_{i}-n_{i}^{\mathrm{Al}}\right)+V_{i}^{\mathrm{AlVa}} n_{i}^{\mathrm{Al}}\right. \\
& \left.-V_{i}^{\mathrm{TiAl}}\left(z_{i}-n_{i}^{\mathrm{Al}}\right)\right] .
\end{aligned}
$$

Here, $z_{i}$ is the coordination number of the $i$ th shell and $n_{i}^{\mathrm{Al}}$ the number of $\mathrm{Al}$ atoms at this shell, and the index "(2)" for pair interaction has been omitted. A similar expression can be obtained for removing a Ti atom (creating a Ti vacancy). So, the slopes of the $\mathrm{Al}$ and Ti vacancy formation energies with respect to $n_{i}^{\mathrm{Al}}$ (under the condition that $n_{j}^{\mathrm{Al}}$ is fixed for $j \neq i$ ), i.e., the change of the formation energies due to increasing $n_{i}^{\mathrm{Al}}$ by one, are

$$
\begin{aligned}
\Delta E^{\mathrm{Al}} & =\frac{1}{2}\left[V_{i}^{\mathrm{TiVa}}-V_{i}^{\mathrm{AlVa}}-V_{i}^{\mathrm{TiAl}}\right] \\
& =-V_{i}^{\mathrm{TiVa}[\mathrm{Al}]}, \\
\Delta E^{\mathrm{Ti}} & =\frac{1}{2}\left[V_{i}^{\mathrm{TiVa}}-V_{i}^{\mathrm{AlVa}}+V_{i}^{\mathrm{TiAl}}\right] \\
& =V_{i}^{\mathrm{AlVa}[\mathrm{Ti}]} .
\end{aligned}
$$

This is a quite interesting result (at least it is not completely intuitive): the difference of the slopes for the $\mathrm{Al}$ and Ti vacancies is given just by $V_{i}^{\mathrm{TiAl}}$, i.e., it is not related to the vacancy-metal effective interactions, while, of course, the particular slopes for $\mathrm{Al}$ and $\mathrm{Ti}$ vacancy formation energies depend on these effective interactions. 
In Fig. 4, we show the effective pair interactions for different pairs of alloy components in $\left(\mathrm{Ti}_{0.49} \mathrm{Al}_{0.49} \mathrm{Va}_{0.02}\right) \mathrm{N}$ calculated with the SGPM implemented within the EMTOCPA technique [22] using the screening constants from the ELSGF calculations as has been described above. First of all, the effective pair Ti-Al interactions at the first two coordination shells are negative and relatively strong, which is just a consequence of the well-known phase separation nature of (Ti-Al)N alloys. At the same time, the vacancy-metal interactions at the first two coordination shells are positive and much stronger than the Ti-Al interactions, so vacancies prefer to be surrounded by metal atoms at the first two coordination shells (or repel each other).

We can now estimate the dependence of the $\mathrm{Ti}$ and $\mathrm{Al}$ vacancy formation energies on the number of $\mathrm{Al}$ atoms at the first and second (which should be large) coordination shells, $n_{1}^{\mathrm{Al}}$ and $n_{2}^{\mathrm{Al}}$, from the effective pair interactions in Fig. 4. First of all, since the difference $V_{1}^{\mathrm{TiVa}}-V_{1}^{\mathrm{AlVa}}$ is approximately equal to $-V_{1}^{\text {TiAl }}$, the Ti vacancy formation energy should be almost independent on the number of $\mathrm{Al}$ (or $\mathrm{Ti}$ ) atoms at the first coordination shell, which is indeed the case of the unrelaxed calculations in Fig. 3. At the same time, the slope of the $\mathrm{Al}$ unrelaxed vacancy formation energies is about 10 mRy or $0.13 \mathrm{eV}$, which is approximately $-V_{1}^{\mathrm{TiAl}}$.

In the case of the second coordination shell, the situation is the opposite. First of all, $V_{2}^{\mathrm{TiVa}}-V_{2}^{\mathrm{AlVa}}$ is negative and $V_{2}^{\mathrm{TiAl}}$ is negative too. This leads to a quite pronounced decrease of the Ti vacancy formation energy with $n_{2}^{\mathrm{Al}}$. The dependence of the $\mathrm{Al}$ vacancy formation energy on $n_{2}^{\mathrm{Al}}$ is similar but weaker than in the case of Ti vacancies. A strong dependence of the $\mathrm{Al}$ and $\mathrm{Ti}$ vacancy formation energies on the number of $\mathrm{Al}$ atoms at the second coordination shell was observed and discussed by Tasnadi et al. [7].

Note that the results presented above are valid only for the unrelaxed supercell calculations and thus, for instance, they cannot be directly compared to the vacancy formation energies in Ref. [7], which are for relaxed structures. One can also see in Fig. 1 that the inclusion of local lattice relaxations leads, first of all, to increasing dispersion of the results for a particular number of $\mathrm{Al}$ atoms at the first coordination shell and, additionally, to a change from the approximately linear dependence on $n_{1}^{\mathrm{Al}}$ to a nonlinear one. The nonlinear dependence on $n_{1}^{\mathrm{Al}}$ actually indicates that the contribution from multisite strain-induced interactions becomes large and important. An accurate consideration of these interactions requires the use of a cluster expansion and it is beyond the scope of the present investigation.

We have also calculated three- and four-site SGPM interactions of the Hamiltonian (2). The strongest three-site interaction is for the triangle formed by two nearest neighbor and one next nearest neighbor sides, $V_{112}^{(3) ; \mathrm{TiTiAl}(\mathrm{Va})}=2.8 \mathrm{mRy}$, and the strongest four-site interaction is for the tetrahedron formed by four nearest neighbor sides and two next nearest neighbor sides: $V_{112211}^{(4) ; T i T i T i(V a)}=5.4 \mathrm{mRy}$. Although they are relatively weak compared with the strongest pair interactions, they still contribute quantitatively to the configurational energetics in some cases.

To demonstrate that the calculated SGPM interactions can quite accurately reproduce the configurational dependence of the $\mathrm{Ti}$ and $\mathrm{Al}$ unrelaxed vacancy formation energies, we cal-

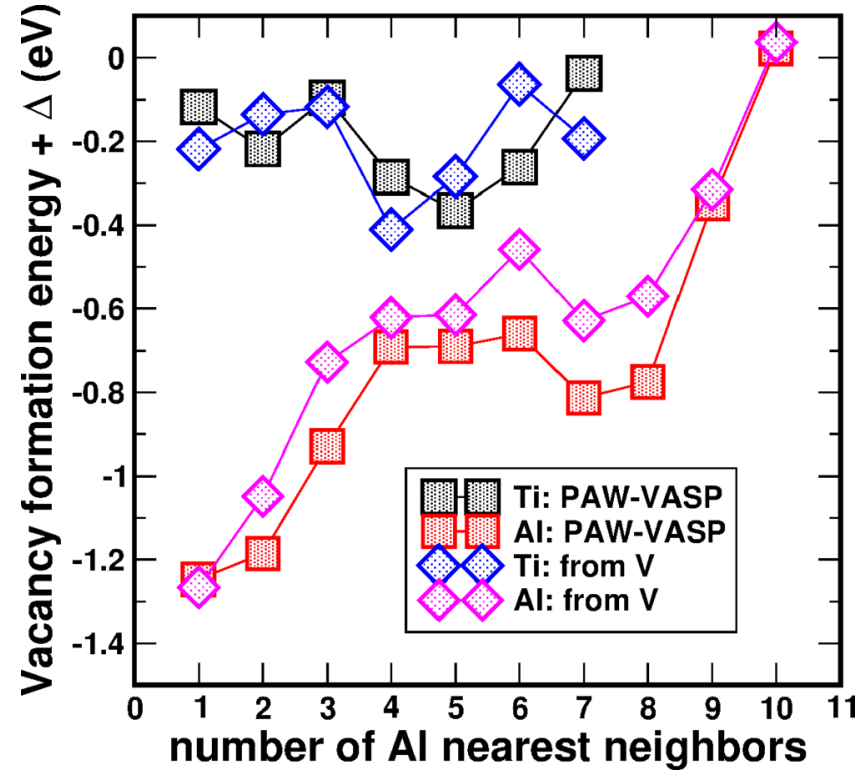

FIG. 5. Unrelaxed $\mathrm{Al}$ and $\mathrm{Ti}$ vacancy formation energies (up to a constant) in $\left(\mathrm{Ti}_{0.5} \mathrm{Al}_{0.5}\right) \mathrm{N}$ as a function of the number of $\mathrm{Al}$ atoms in the first coordination shell obtained in the direct 512-atom supercell calculations by PAW-VASP (no local lattice relaxations) and from the SGPM effective interactions of the configurational Hamiltonian. All sets of energies are determined up to a constant.

culate a set of $\mathrm{Ti}$ and $\mathrm{Al}$ vacancy formation energies on a fixed lattice (without contribution from local lattice relaxations) using a relatively large 512-atom $[4 \times 4 \times 4(\times 8)]$ supercell with random distribution of $\mathrm{Ti}$ and $\mathrm{Al}$ atoms according to atomic short-range order parameters up to the eighth fcc coordination shell. Such a large supercell allows us to find a wide spectrum of atomic distribution correlation functions for $\mathrm{Ti}$ and $\mathrm{Al}$ sites.

The results of supercell PAW-VASP calculations are shown in Fig. 5 for different $n_{1}^{\mathrm{Al}}$. One should bear in mind, however, that the local environments of the $\mathrm{Al}$ and Ti sites for different $n_{1}^{\mathrm{Al}}$ differ not only by $n_{1}^{\mathrm{Al}}$, but the whole spectrum of the atomic distribution correlation functions. Therefore, in order to get the configurational energy contribution from the Hamiltonian (2) to the vacancy formation energy, we first determined the set of the corresponding two-, three-, and four-site correlation functions for a given choice of a vacant site in the supercell and then calculated the relative (up to a constant) value of the vacancy formation energy from the SGPM interactions.

In these calculations we have used the first 10 effective pair interactions, and the 10 and three strongest effective three-site and four-site interactions, respectively. As one can see, the agreement between direct PAW-VASP and SGPM results is very good considering that these are totally different methods. However, it is a relatively easy case of unrelaxed configurational dependences. In the case of relaxed calculations, an accurate investigation of configurational effects requires the use of the cluster expansion method and very large supercell calculations to converge the effective interactions (including strain-induced interactions). Such an investigation is very resource requiring and beyond the present investigation. 
TABLE I. Vacancy formation energy (in $\mathrm{eV}$ ) for the sites of $\mathrm{Al}$ and $\mathrm{Ti}$ atoms at the (001) (Ti,Al)N/AlN interface.

\begin{tabular}{|c|c|c|c|}
\hline Metal & Number of Al NN & Unrelaxed & Relaxed \\
\hline \multicolumn{4}{|c|}{$\left(\mathrm{Ti}_{0.5} \mathrm{Al}_{0.5}\right) \mathrm{N}$} \\
\hline $\mathrm{Ti}$ & 7 & 1.455 & 2.466 \\
\hline $\mathrm{Ti}$ & 9 & 1.526 & 2.208 \\
\hline $\mathrm{Ti}$ & 10 & 1.826 & 2.772 \\
\hline $\mathrm{Al}$ & 7 & 0.693 & 1.915 \\
\hline $\mathrm{Al}$ & 9 & 0.753 & 1.800 \\
\hline $\mathrm{Al}$ & 10 & 1.064 & 1.895 \\
\hline \multicolumn{4}{|c|}{ AlN } \\
\hline $\mathrm{Al}$ & 9 & 5.102 & 1.647 \\
\hline $\mathrm{Al}$ & 11 & 6.002 & 2.086 \\
\hline
\end{tabular}

\section{Metal vacancies at (Ti,Al)N/AIN interface}

Although a complete picture with atomically resolved information about $\mathrm{Ti}$ and $\mathrm{Al}$ distribution in $(\mathrm{Ti}, \mathrm{Al}) \mathrm{N}$ films is absent, recent detailed structural information [33] indicates that the composition of nanolamellae $(\mathrm{Ti}, \mathrm{Al}) \mathrm{N}$ films is quite inhomogeneous and spatially complicated on a nanometer scale. Such structures are practically impossible to model in $a b$ initio calculations. Instead, one can obtain results for an "idealized" interface of two (Ti,Al)N alloys with different composition, similar to the interface of the nanolamellae structures.

Here, we choose the simplest (for modeling and interpretation) possible system, but still to some extent relevant: the (001) interface between random $\left(\mathrm{Ti}_{0.5} \mathrm{Al}_{0.5}\right) \mathrm{N}$ alloy and $\mathrm{AlN}$. It is modeled by a 288 -atom $[3 \times 3 \times 4(\times 8)]$ supercell, half of which is a random $\left(\mathrm{Ti}_{0.5} \mathrm{Al}_{0.5}\right) \mathrm{N}$ alloy and the other half $\mathrm{AlN}$. The lattice constant was chosen to be $4.11 \AA$. The shape of the supercell and its volume were fixed and only local lattice relaxations were allowed. According to our test calculations, the effect of shape relaxation on the finite result for vacancy formation energies was small, about $0.05 \mathrm{eV}$.

The calculated vacancy formation energies are presented in Table I. As one can see, both Ti and Al vacancy formation energies increase with the number of $\mathrm{Al}$ nearest neighbors in $\left(\mathrm{Ti}_{0.5} \mathrm{Al}_{0.5}\right) \mathrm{N}$. However, such a dependence disappears when local lattice relaxations are allowed. The increasing value of the vacancy formation energies with the account of local lattice relaxations is most probably due to a large gain of energy due to local lattice relaxations in the system without vacancy.

At the same time, there is apparently a huge drop of the initial unrelaxed Al vacancy formation energies at the interface in AlN after local lattice relaxations are allowed. So, the initial values, which are similar to those in AlN, become close those in the case $\left.\mathrm{Ti}_{0.5} \mathrm{Al}_{0.5}\right) \mathrm{N}$. The reason for that large decrease of the formation energies is probably due to the fact that the bonding in AlN becomes of a metal character which provides additional possibility for local relaxations.

These results show that in inhomogeneous alloys, like lamella structures, the vacancy formation energy can vary substantially. The point is that $\left(\mathrm{Ti}_{0.4-0.1} \mathrm{Al}_{0.6-0.9}\right) \mathrm{N}$ alloys are the least stable among all (Ti-Al)N alloys [10]. Although the film formations during PVD or CVD are nonequilibrium processes, strong thermodynamic driving forces can contribute to create locally inhomogeneous regions where vacancy formation energies can exhibit large fluctuations. This is especially important considering the fact that such vacancies should be quite mobile during the growth process due to, e.g., enhanced diffusion at the surface. A proper theoretical modeling of these effects is a very important and interesting task, but requires the use of huge computational resources and is beyond the aims of the present investigation.

\section{SUMMARY}

(Ti-Al)N alloys are used as coatings and can be produced by PVD or CVD techniques and they can contain a large amount of structural vacancies, which play an important role in the stability of the grown films. Here, we have investigated the energetics of nitrogen and metal vacancies at several (TiAl)N-alloy compositions and in different local environments in ab initio calculations.

PAW-VASP calculations show an increase of the average $\mathrm{N}$ vacancy formation energy both with $\mathrm{Al}$ concentration and number of $\mathrm{Al}$ atoms at the first coordination shell. The nitrogen vacancy formation energies are relatively large in the whole concentration range, and our results are in good agreement with the previous ab initio calculations by Euchner and Mayrhofer [6].

At the same time, the concentration dependence of the formation energies of metal vacancies is at variance with the results of Euchner and Mayrhofer [6] for Al-rich compositions: according to our results the vacancy formation energies of $\mathrm{Al}$ and $\mathrm{Ti}$ drop to very low values, especially $\mathrm{Al}$ vacancy formation energies, while they steadily grow in calculations by Euchner and Mayrhofer. The main difference between our calculations and those in Ref. [6] is the size of the supercell, which is significantly larger in our case.

To elucidate the dependence of the formation energies of $\mathrm{Ti}$ and $\mathrm{Al}$ on their local environment we separately calculated these energies with and without account for local lattice relaxations. The latter has allowed us to use an analysis in terms of the standard (concentration dependent) Ising Hamiltonian with SGPM interactions obtained in EMTO-CPA calculations. We showed that this interaction model can produce an almost quantitatively accurate description for the local configurational dependence of the vacancy formation energies.

Moreover, it allows one to understand that the nonlinear dependence of the vacancy formation energies on the number of $\mathrm{Al}$ (or $\mathrm{Ti}$ ) atoms should be related to multisite interactions for clusters which have at least two nearest neighbor sites connected to the given vacant site. This is clearly the case of relaxed vacancy formation energies. The origin of strong multisite (most probably three-site) stain-induced interactions in this particular alloy is related to the covalentlike type of bonding, which is very sensitive to the change of bond angles.

Finally, we have also calculated the vacancy formation energies at the (001) (Ti,Al)N/AlN interface. Here, the relaxation energy contribution appears to be especially strong in $\mathrm{AlN}$, about $4 \mathrm{eV}$, which brings down the $\mathrm{Al}$ formation energy close to the values in $\left(\mathrm{Ti}_{0.5} \mathrm{Al}_{0.5}\right) \mathrm{N}$. These results show that the vacancy formation energy can be quite sensitive not only to the composition and local environment, but also to specific spatial composition changes, like at the interfaces. The latter is 
a very interesting and important subject for further modeling, which can shed more light on what happens to vacancies during $(\mathrm{Ti}, \mathrm{Al}) \mathrm{N}$ film growth and in their exploitation.

\section{ACKNOWLEDGMENTS}

The DFT simulations were performed on resources provided by the Swedish National Infrastructure for Computing (SNIC) at PDC (Stockholm) and NSC (Linköping) and using the DECI resource Archer based in UK at EPCC with support from PRACE. This project was financially supported by the Swedish Foundation for Strategic Research via SSF Contract No. RMA15-0048. A.V.R. acknowledges a European Re- search Council grant, the VINNEX center Hero-m, financed by the Swedish Governmental Agency for Innovation Systems (VINNOVA), Swedish industry, and the Royal Institute of Technology (KTH). A.V.R. also gratefully acknowledges the financial support under the scope of the COMET program within the K2 Center "Integrated Computational Material, Process and Product Engineering (IC-MPPE)" (Project No. 859480). This program is supported by the Austrian Federal Ministries for Climate Action, Environment, Energy, Mobility, Innovation and Technology (BMK) and for Digital and Economic Affairs (BMDW), represented by the Austrian research funding association (FFG), and the federal states of Styria, Upper Austria, and Tyrol.
[1] J.-E. Sundgren, Thin Solid Films 128, 21 (1985).

[2] H. E. Rebenne and D. G. Bhat, Surf. Coat. Technol. 63, 1 (1994).

[3] S. PalDey and S. Deevi, Mater. Sci. Eng.: A 342, 58 (2003).

[4] M. to Baben, M. Hans, D. Primetzhofer, S. Evertz, H. Ruess, and J. M. Schneider, Mater. Res. Lett. 5, 158 (2016).

[5] I. Endler, M. Höhn, M. Herrmann, R. Pitonak, S. Ruppi, M. Schneider, H. van den Berg, and H. Westphal, Surf. Coat. Technol. 203, 530 (2008).

[6] H. Euchner and P. Mayrhofer, Surf. Coat. Technol. 275, 214 (2015).

[7] F. Tasnádi, A. V. Lugovskoy, M. Odén, and I. A. Abrikosov, Mater. Des. 114, 484 (2017).

[8] P. A. Korzhavyi, L. V. Pourovskii, H. W. Hugosson, A. V. Ruban, and B. Johansson, Phys. Rev. Lett. 88, 015505 (2001).

[9] S. Yu, Q. Zeng, A. R. Oganov, G. Frapper, and L. Zhang, Phys. Chem. Chem. Phys. 17, 11763 (2015).

[10] B. Alling, A. Karimi, L. Hultman, and I. Abrikosov, Appl. Phys. Lett. 92, 071903 (2008).

[11] P. E. Blöchl, Phys. Rev. B 50, 17953 (1994).

[12] G. Kresse and D. Joubert, Phys. Rev. B 59, 1758 (1999).

[13] G. Kresse and J. Hafner, Phys. Rev. B 48, 13115 (1993).

[14] G. Kresse and J. Furthmüller, Comput. Mater. Sci. 6, 15 (1996).

[15] J. P. Perdew, K. Burke, and M. Ernzerhof, Phys. Rev. Lett. 77, 3865 (1996).

[16] H. J. Monkhorst and J. D. Pack, Phys. Rev. B 13, 5188 (1976).

[17] F. Ducastelle and F. Gautier, J. Phys. F 6, 2039 (1976).

[18] F. Ducastelle, Order and Phase Stability in Alloys, Cohesion and Structure Vol. 3 (North-Holland, Amsterdam: sole distributors for the USA and Canada, Elsevier Science Pub. Co., New York, 1991).

[19] A. V. Ruban and H. L. Skriver, Phys. Rev. B 66, 024201 (2002).

[20] A. V. Ruban, S. I. Simak, P. A. Korzhavyi, and H. L. Skriver, Phys. Rev. B 66, 024202 (2002).

[21] A. V. Ruban, S. Shallcross, S. I. Simak, and H. L. Skriver, Phys. Rev. B 70, 125115 (2004).

[22] A. V. Ruban and M. Dehghani, Phys. Rev. B 94, 104111 (2016).

[23] O. K. Andersen, O. Jepsen, and G. Krier, Lectures on Methods of Electronic Structure Calculations (World Scientific, Singapore, 1994), p. 63.

[24] L. Vitos, Computational Quantum Mechanics for Materials Engineers, The EMTO Method and Applications (Springer-Verlag, London, 2007).

[25] P. Soven, Phys. Rev. 156, 809 (1967).

[26] B. L. Gyorffy, Phys. Rev. B 5, 2382 (1972).

[27] L. Vitos, I. A. Abrikosov, and B. Johansson, Phys. Rev. Lett. 87, 156401 (2001)

[28] I. A. Abrikosov, A. M. N. Niklasson, S. I. Simak, B. Johansson, A. V. Ruban, and H. L. Skriver, Phys. Rev. Lett. 76, 4203 (1996).

[29] I. A. Abrikosov, S. I. Simak, B. Johansson, A. V. Ruban, and H. L. Skriver, Phys. Rev. B 56, 9319 (1997).

[30] O. E. Peil, A. V. Ruban, and B. Johansson, Phys. Rev. B 85, 165140 (2012).

[31] J. P. Perdew and Y. Wang, Phys. Rev. B 45, 13244 (1992).

[32] A. V. Ruban and H. L. Skriver, Phys. Rev. B 55, 856 (1997).

[33] R. Qiu, H. Aboulfadl, O. Bäcke, D. Stiens, H.-O. Andrén, and M. Halvarsson, Surf. Coat. Technol. 426, 127741 (2021). 